Supplement of

\title{
Bulk and Molecular-Level Characterization of Laboratory-Aged Biomass Burning Organic Aerosol from Oak Leaf and Heartwood Fuels
}

Claire F. Fortenberry, et al.

Correspondence to: Brent J. Williams (brentw@wustl.edu) 


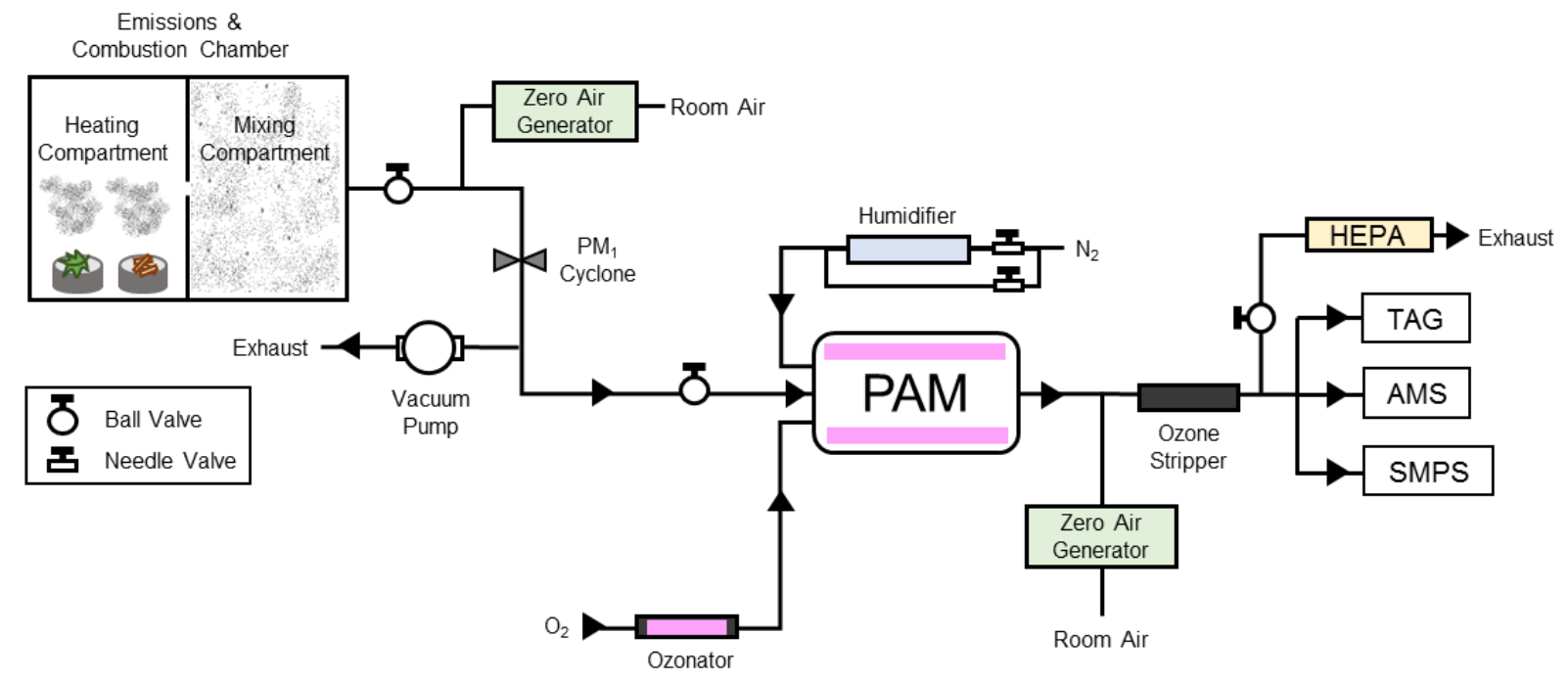

Figure S1: Flow diagram of experimental setup

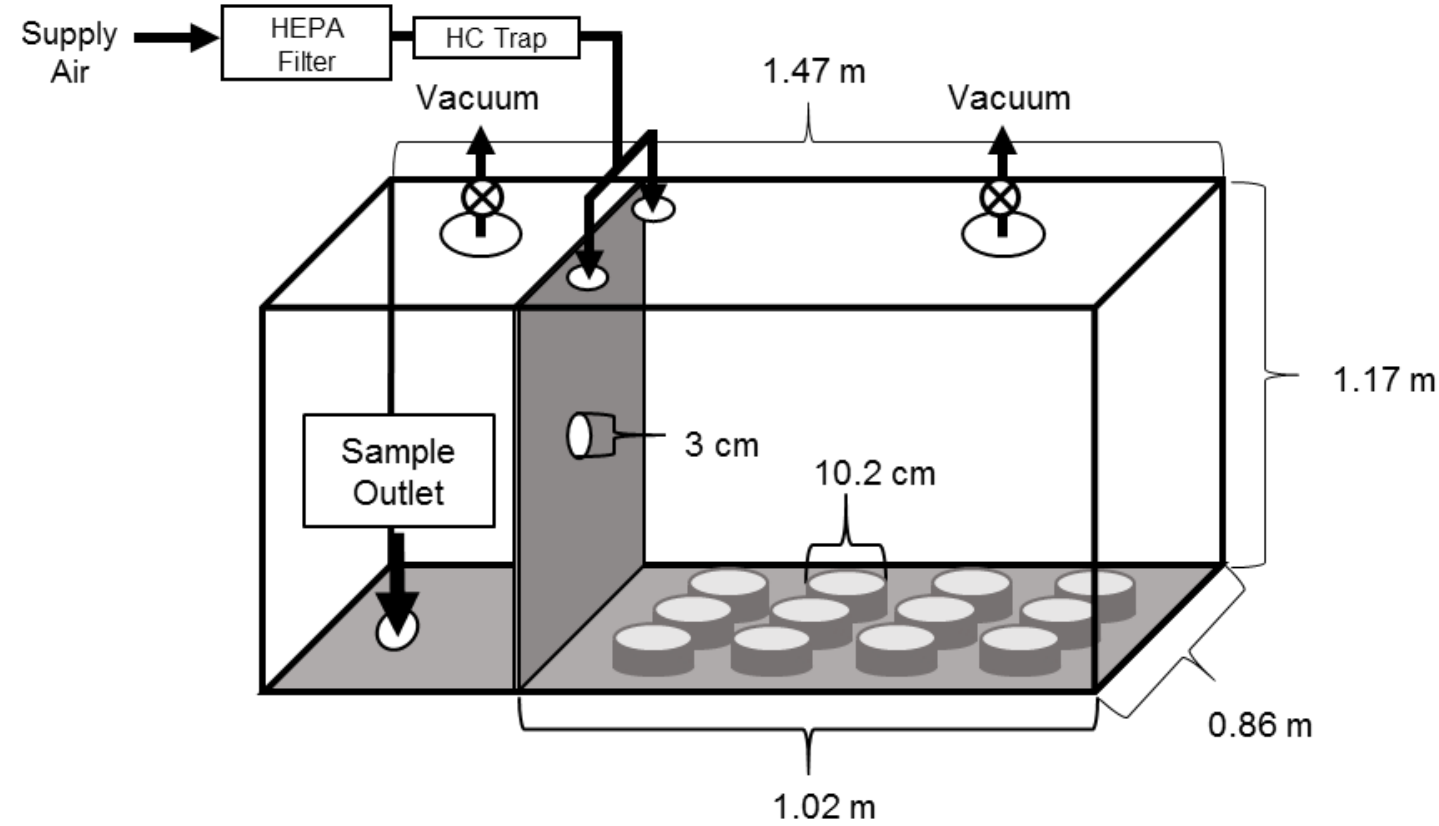

Figure S2: Diagram of emissions and combustion chamber. 


\section{Method: PAM Calibrations}

The PAM was calibrated a week prior to the first experimentation period. Equivalent atmospheric aging times were determined by calibrating the PAM reactor with sulfur dioxide $\left(\mathrm{SO}_{2}\right)$ gas, as described by Kang, et al (Kang et al., 2007). A constant stream of $\mathrm{SO}_{2}$ (Airgas, Inc., Radnor, PA) was introduced into the PAM chamber at a steady flow rate of $0.02 \mathrm{~L} \mathrm{~min}^{-1}$. Humidified $\mathrm{N}_{2}(\mathrm{RH}=30 \%)$ and $\mathrm{O}_{2}$ flow rates were maintained at $4.6 \mathrm{~L} \mathrm{~min}^{-1}$ and $0.4 \mathrm{~L} \mathrm{~min}^{-1}$, respectively. The total flow rate through the PAM chamber was maintained at $10 \mathrm{~L} \mathrm{~min}^{-1}$ throughout the calibration. The PAM ultraviolet (UV) lamp voltage was systematically varied from 30 to $100 \mathrm{~V}$, and the $\mathrm{SO}_{2}$ concentration was measured for 25 minutes at each setting using an $\mathrm{SO}_{2}$ analyzer (Model 43i-TLE, Thermo Fischer Scientific, Waltham, $\mathrm{MA})$. $\mathrm{OH} \cdot$ exposure $\left(\mathrm{OH}_{\text {exp }}\right)$, defined as the $\mathrm{OH} \cdot$ concentration multiplied by the reactor residence time, was calculated based on a pseudo-first order rate expression for the reaction of $\mathrm{SO}_{2}$ with $\mathrm{OH} \cdot$ :

$\frac{d\left[\mathrm{SO}_{2}\right]}{d t}=-k_{O H}[\mathrm{OH}]\left[\mathrm{SO}_{2}\right]$

$\mathrm{OH}_{\exp }=[\mathrm{OH}] t=\frac{1}{k_{O H}} \ln \left(\frac{\left[\mathrm{SO}_{2}\right]_{0}}{\left[\mathrm{SO}_{2}\right]}\right)$

where $k_{O H}$ is the rate constant for the reaction of $\mathrm{SO}_{2}$ with $\mathrm{OH} \cdot\left(9 \times 10^{-13} \mathrm{~cm}^{3}\right.$ molec $^{-1} \mathrm{~s}^{-1}$; Davis et al., 1979; Lambe et al., 2011) and $\left[\mathrm{SO}_{2}\right]_{0}$ is the initial $\mathrm{SO}_{2}$ concentration.

Equivalent aging times $\left(t_{\text {equiv }}\right)$ were determined for each voltage setting assuming an average atmospheric $\mathrm{OH}$. concentration $\left([\mathrm{OH}]_{\mathrm{atm}}\right.$ ) of $1.5 \times 10^{6} \mathrm{molec}^{-3}$ (Mao et al., 2009) and a reactor residence time of 78 seconds:

$t_{\text {equiv }}=\frac{[\mathrm{OH}]_{\mathrm{atm}}}{\mathrm{OH}_{\exp }}$

To obtain $\mathrm{OH}_{\text {exp }}$ and equivalent aging times for each PAM UV lamp setting, the results of the calibration were fit with a linear regression $\left(r^{2}=0.957\right) . \mathrm{OH}_{\mathrm{exp}}$ and equivalent aging time values are given as functions of the PAM light voltage in Figure S3a.

$\mathrm{OH} \cdot$ forms in the PAM reactor when monatomic oxygen radicals, produced as a result of $\mathrm{O}_{3} \mathrm{UV}(254 \mathrm{~nm})$ photolysis, react with water molecules (Ehhalt et al., 1990; Lambe et al., 2011; Levy, 1971; Palm et al., 2016; Shetter et al., 1996). Previous PAM studies have demonstrated that $\mathrm{OH} \cdot$ production increases with increasing relative humidity (RH) (Kang et al., 2007; Li et al., 2015; Peng et al., 2015, 2016). The sensitivity of $\mathrm{OH}_{\text {exp }}$ to $\mathrm{RH}$ in our PAM reactor was investigated by performing $\mathrm{SO}_{2}$ calibrations at $10 \%, 20 \%$, and $30 \% \mathrm{RH}$ (Figure $\mathrm{S} 3 \mathrm{~b}$ ). 

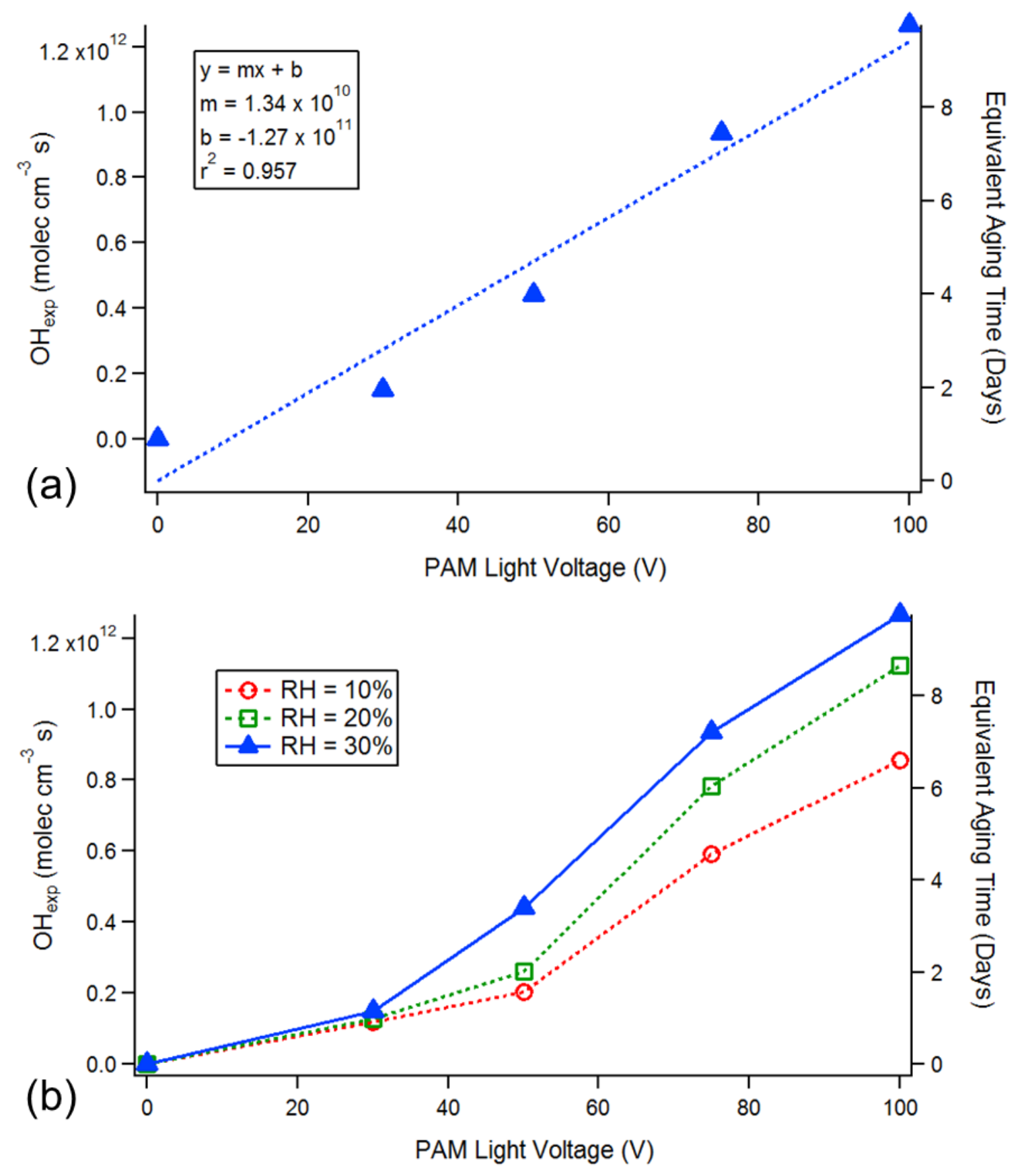

Figure S3. Results from PAM reactor $\mathrm{SO}_{2}$ calibrations, displayed as $\mathrm{OH}_{\text {exp }}$ and equivalent aging times as functions of PAM reactor light voltage. (a) results from the calibration at $\mathrm{RH}=30 \%$, with linear regression parameters provided; (b) results from calibrations at 10\% (red circles), 20\% (green squares), and 30\% (blue triangles) RH. 


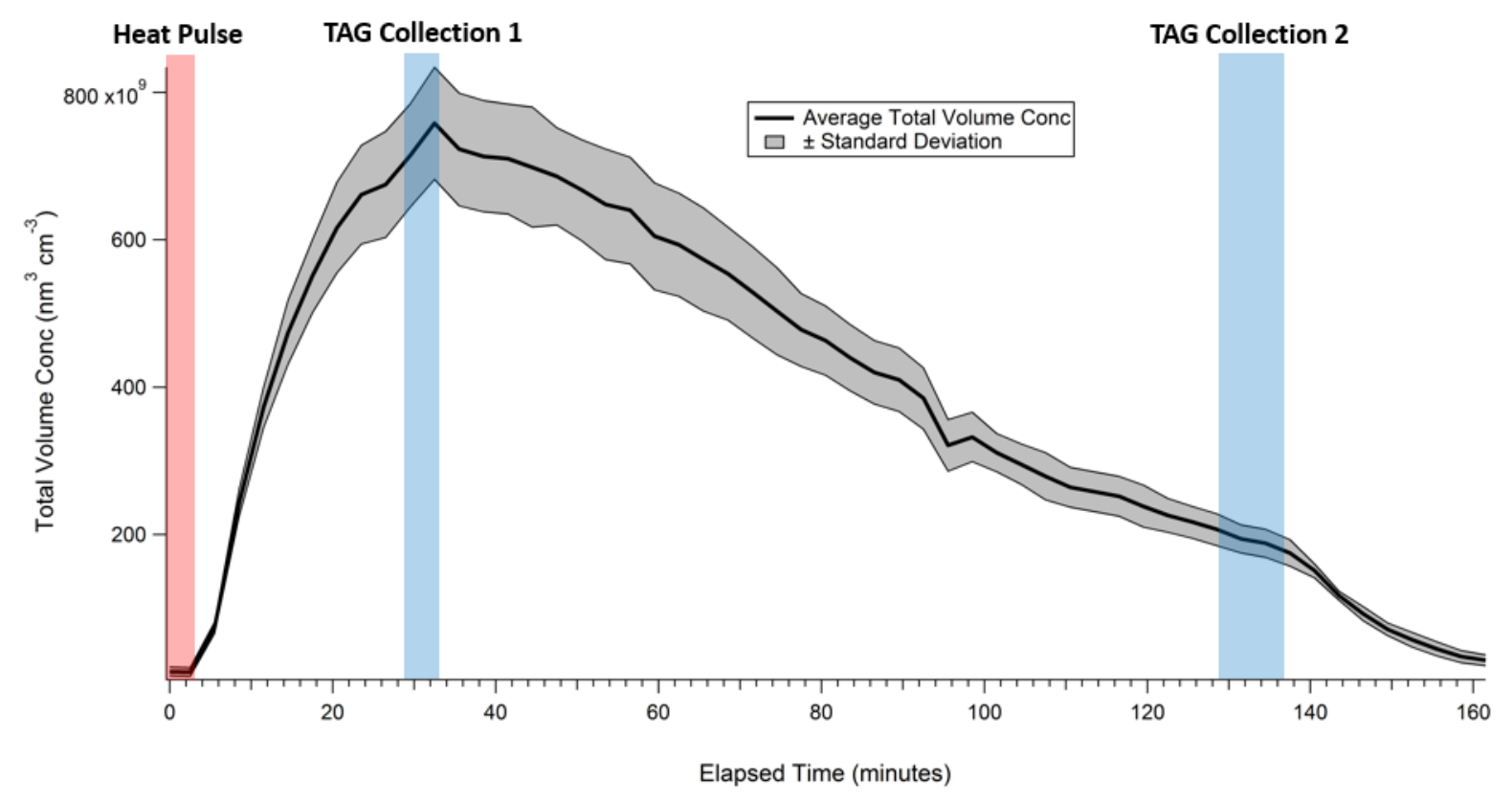

Figure S4. SMPS total volume concentration $\left(\mathrm{nm}^{3} \mathrm{~cm}^{-3}\right)$ over time for oak heartwood $\mathrm{PM}_{1}$ at 3.4 days of equivalent aging, presented as an average of triplicate measurements (bold black trace) \pm one standard deviation (gray shaded region). The heat pulse occurred during the period encompassed by the red shaded region, beginning at elapsed time $=0$ minutes. All TAG data presented in this work were collected for a duration of 4 minutes beginning 30 minutes after the start of the heat pulse (blue shaded region, "TAG Collection 1"). Additional sample collections (e.g. blue shaded region, "TAG Collection 2"; other subsequent collections not shown) were also obtained to evaluate the cleanliness of the emissions and combustion chamber. 
1

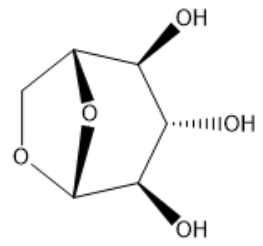

2

3

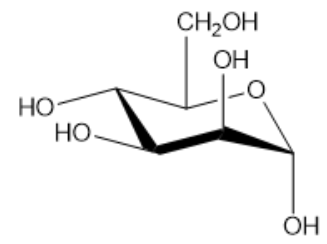

4<smiles>CC(C)(C)CC(C)(C)C(=O)O</smiles>

5<smiles>CCC(C)(C)CC(C)(C)C</smiles>

6<smiles>C=CC(CC)(CC)CC(C)(C)C</smiles>

7

$$
\mathrm{H}_{3} \mathrm{C}-\left(\mathrm{C}^{\mathrm{H}_{2}}\right)_{23} \mathrm{CH}_{3}
$$

8<smiles>CCCC(C=O)CC(C)(C)C</smiles>

9<smiles>CCC(CO)(CCO)CC(C)(C)C</smiles>

10<smiles>[Y20]C(C=O)(C=O)CC(C)(C)C</smiles>

11<smiles>CC(C)(C)CC(C)(C)C</smiles>

12<smiles>CC(C)(C)CC(C=O)C=O</smiles>

13<smiles>CC(C)(C)CC=O</smiles>

14

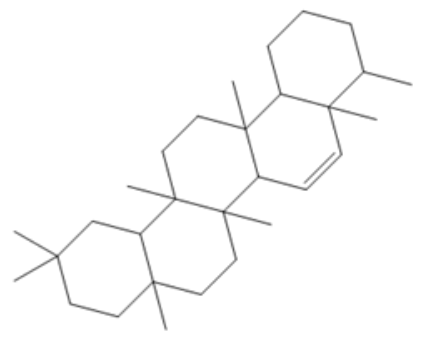

15

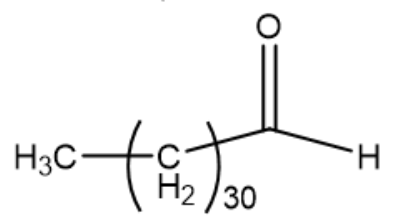

16

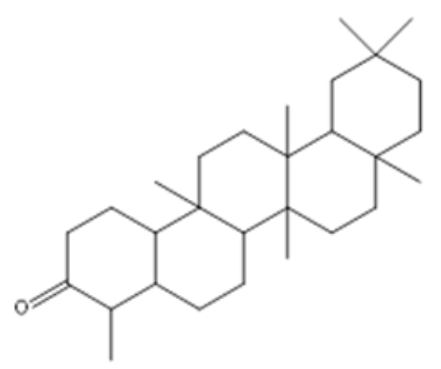

Figure S5: Structures identified in oak leaf BBOA TAG chromatograms. Corresponding compound names are provided in Table S2. 
17<smiles>COc1cccc(OC)c1O</smiles>

18<smiles>COc1cc(C=O)ccc1O</smiles>

19

$$
\text { HO }
$$<smiles>C=C=Cc1ccc(O)c(OC)c1</smiles>

20

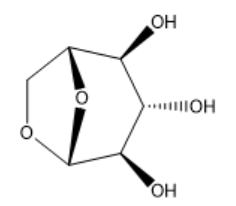

21<smiles>O=C(CO)[C@@H](O)[C@H](O)[C@H](O)[C@H](O)CO</smiles>

22

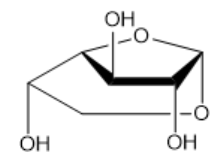

23

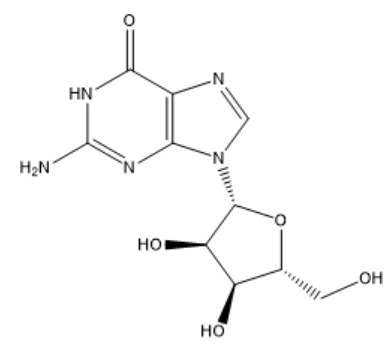

24

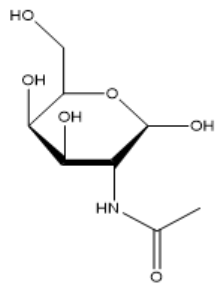

25<smiles>C=Cc1cc(OC)c(O)c(OC)c1</smiles>

26<smiles>COc1cc(C=O)ccc1C</smiles>

27<smiles>CC#Cc1cc(OC)c(O)c(OC)c1</smiles>

28<smiles>COc1cc(C(C)=O)cc(OC)c1O</smiles>

29
30<smiles>COc1cc(CC(C)=O)cc(OC)c1O</smiles>

31

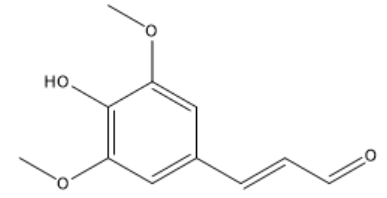

Figure S6: Structures identified in oak wood BBOA TAG chromatograms. Corresponding compound names are provided in Table $\mathrm{S} 3$. 


\section{Method: Oak leaf solvent extractions}

The solvent extraction method used to extract leaf wax components was adapted from a previously published method (Gulz and Boor, 1992). A $52.9 \pm 0.5 \mathrm{mg}$ leaf sample was gently broken into roughly centimeter-wide pieces and added to $2 \mathrm{~mL}$ of a 2:1:1 mixture of chloroform, acetone, and methanol. The leaf sample was submerged in the solvent mixture for one minute under gentle agitation by hand. The leaf was then removed for one minute and submerged again for one minute. Solid leaf components were decanted from the extraction prior to TAG analysis. A $3 \mu \mathrm{L}$ aliquot of the extraction was injected onto the TAG CTD cell through its standard injection port (Kreisberg et al., 2009), desorbed, separated, and analyzed using the previously described TAG thermal desorption and GC oven programs.

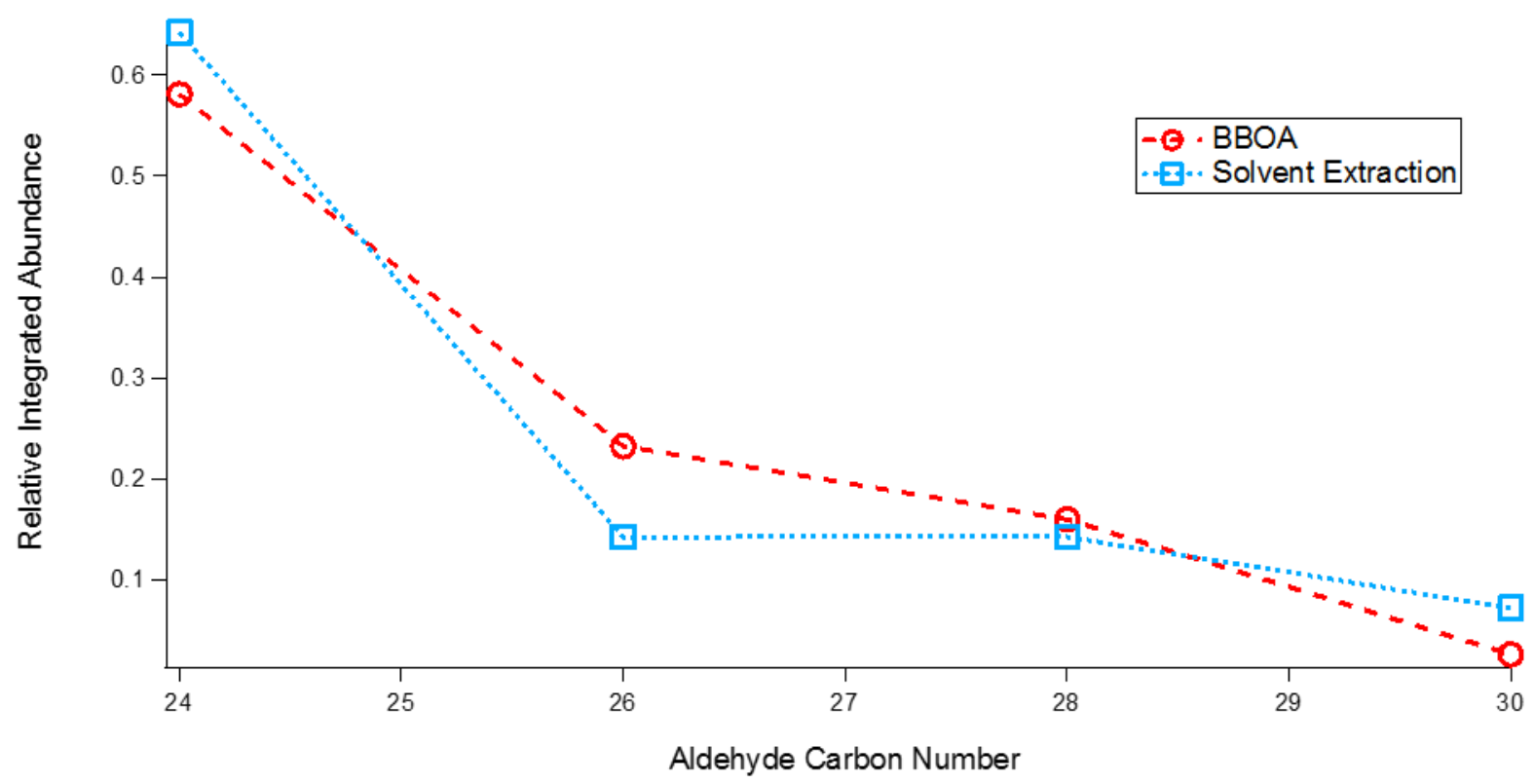

Figure S7. Relative integrated abundances for four different aldehydes measured in a TAG leaf BBOA chromatogram ( 0 days of equivalent aging) and a solvent extraction chromatogram. Each point corresponds to the integrated $\mathrm{m} / \mathrm{z} 82$ abundance normalized to the sum of the integrated $\mathrm{m} / \mathrm{z}, 82$ abundances over the four different aldehydes within the chromatogram. 




Figure S8. One possible mechanism for the formation of vanillin from larger lignin decomposition products within the PAM reactor. In the first stage (R1), a peroxy radical intermediate is formed following $\mathrm{OH} \cdot$-driven hydrogen abstraction from the phenolic substituent, rearrangement, and $\mathrm{HO}_{2}$ radical addition to the beta carbon. In the second stage (R2), the radical intermediate undergoes an intramolecular nucleophilic attack, causing breakage of the $\mathrm{C}_{\alpha}-\mathrm{C}_{\beta}$ bond and resulting in formation of two distinct aldehydes. This mechanism assumes sufficiently high concentrations of the $\mathrm{HO}_{2}$ radical are present within the reactor. The mechanism was adapted from the alkaline oxygen delignification mechanism described by Wong, et al. (Wong et al., 2010). 

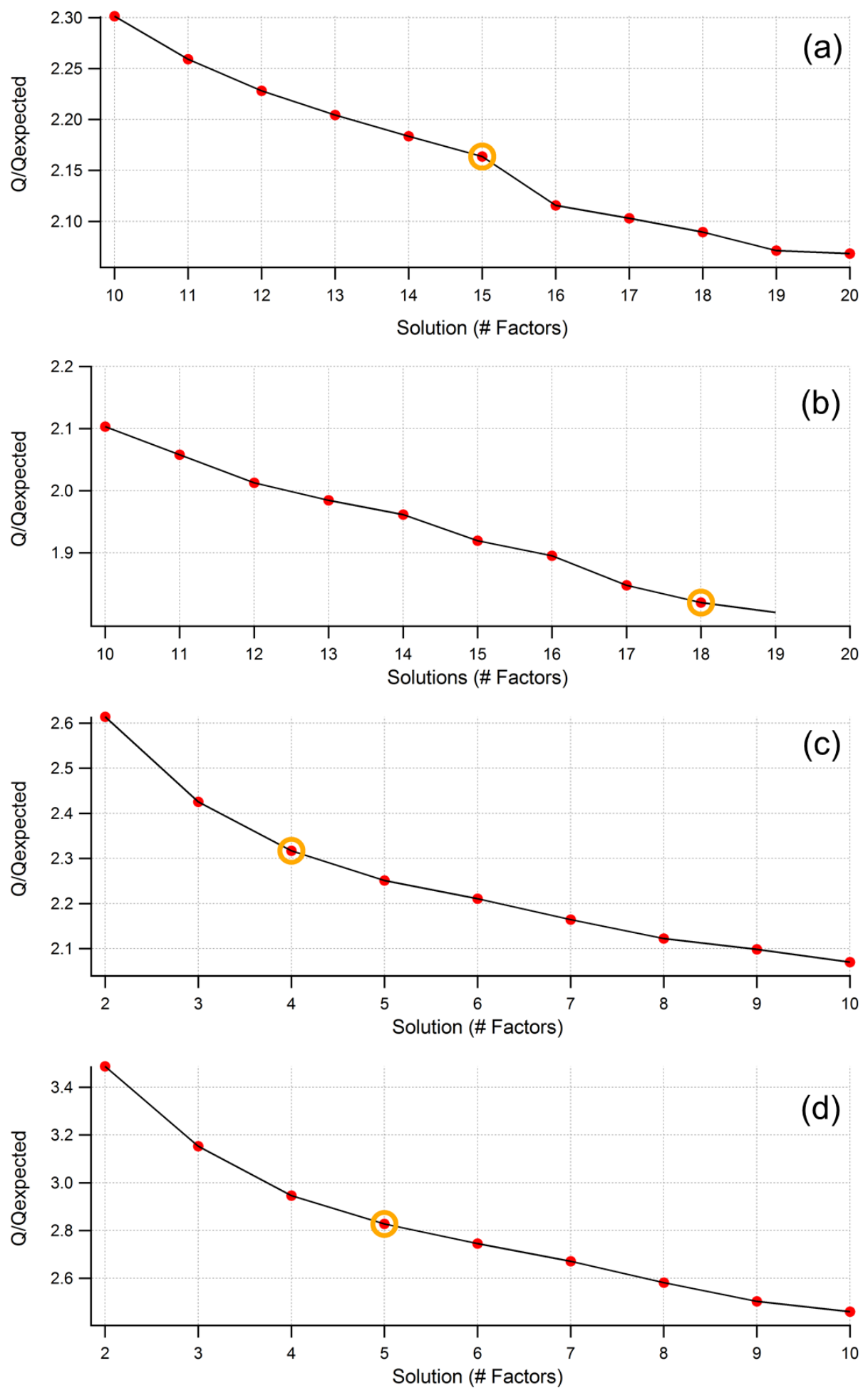

Figure S9. $Q / Q_{\text {exp }}$ as a function of factor number, where the orange circle indicates the number of factors in the chosen solution for PMF calculations on: (a) oak leaf compound window; (b) oak wood compound window; (c) oak leaf decomposition window; (d) oak wood decomposition window. 
(a)

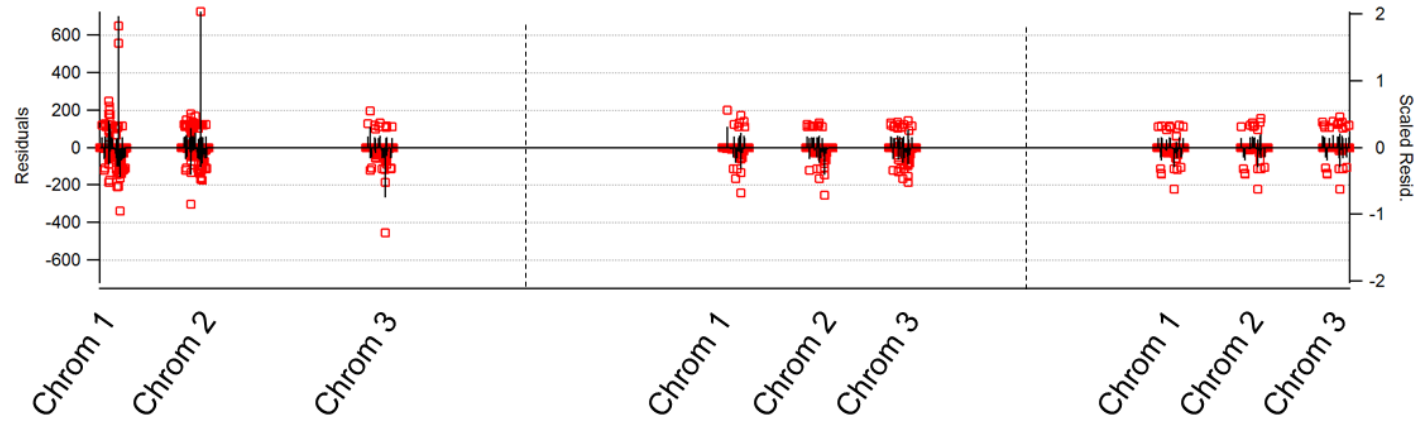

(b)

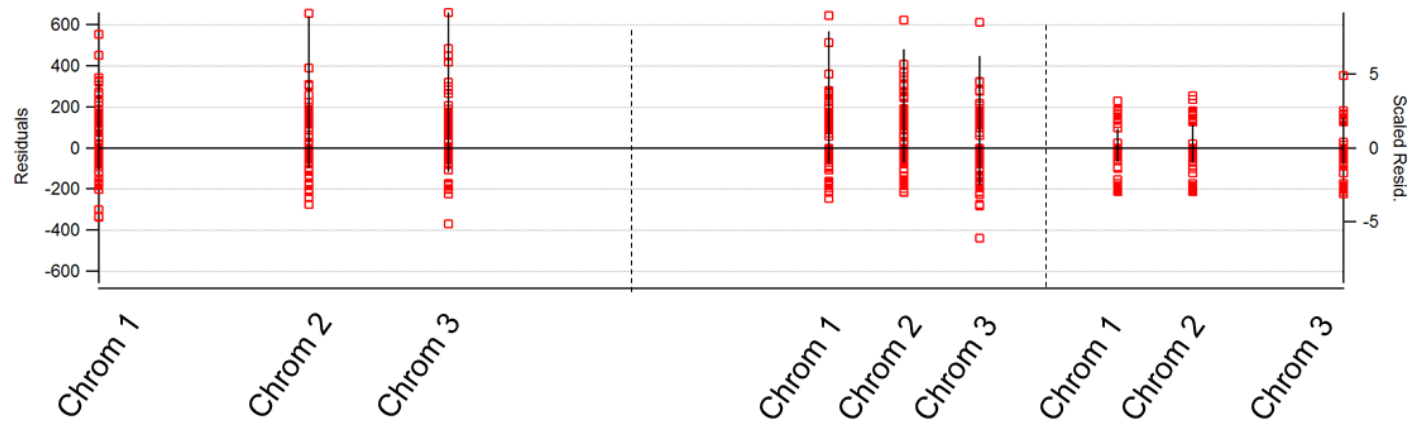

(c)

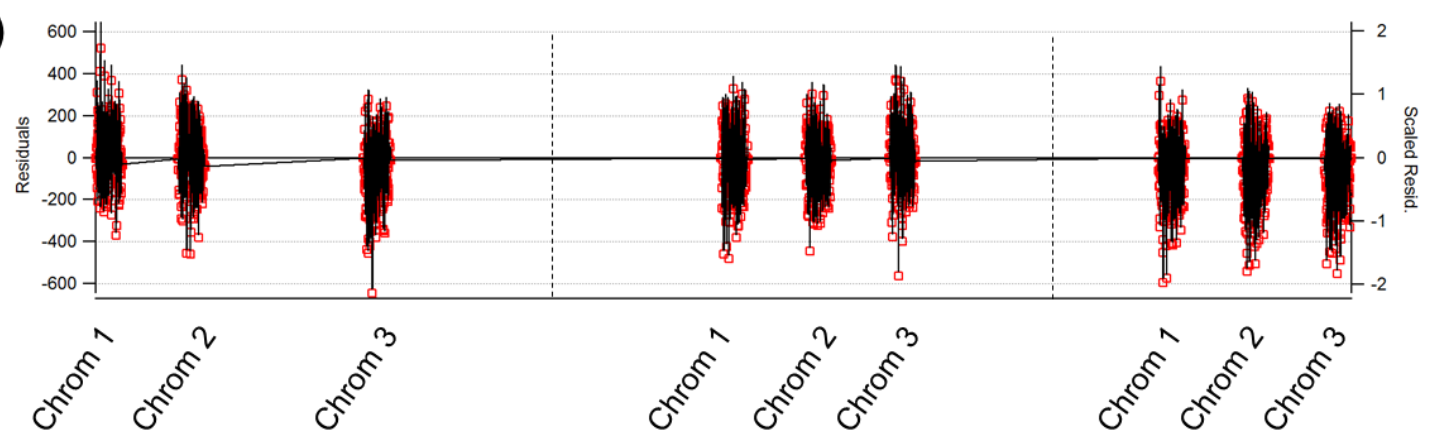

(d)

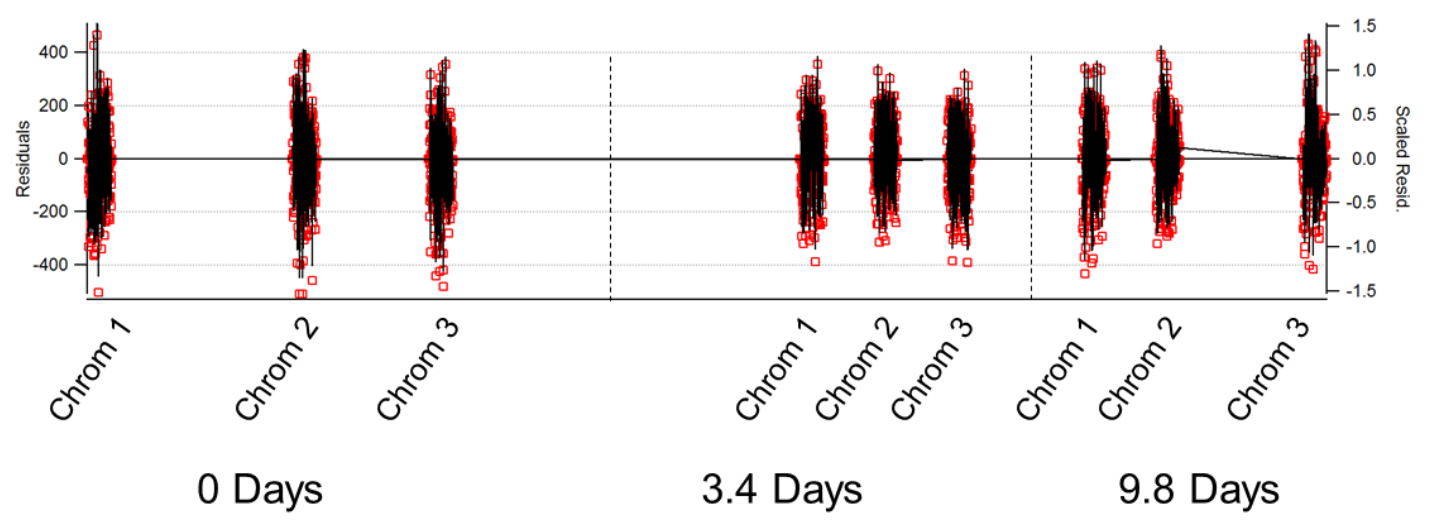

Figure S10. Residuals (black lines) and scaled residuals (red dots) from PMF calculations on: (a) oak leaf compound window; (b) oak wood compound window; (c) oak leaf decomposition window; (d) oak wood decomposition window. 


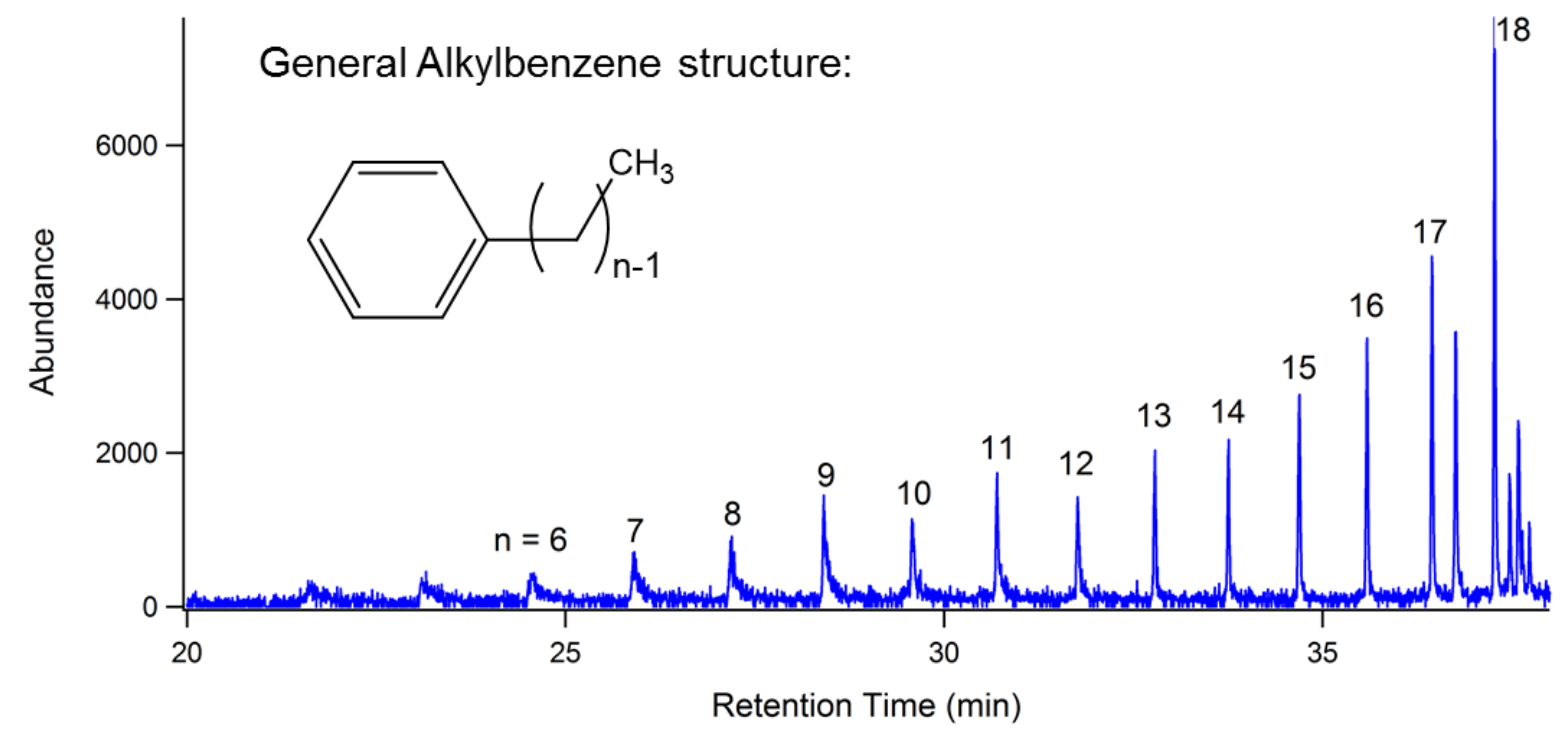

Figure S11. $\mathrm{m} / \mathrm{z}, 92$ single ion chromatogram (SIC) from TAG analysis of oak leaf extract showing a series of alkylbenzenes. The number above each peak denotes the number of carbons ( $n$ ) present in the alkyl chain (e.g. $n=9$ corresponds to nonyl-benzene). 


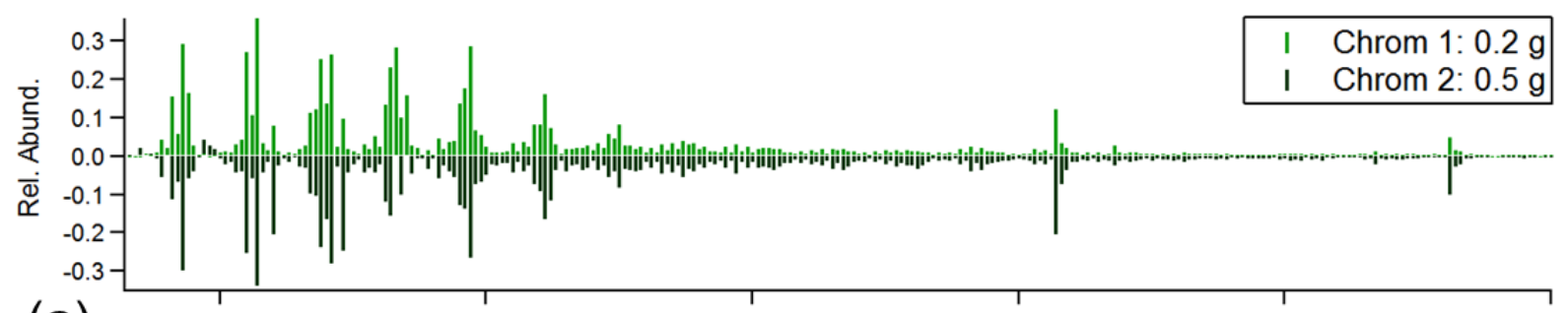

(a)

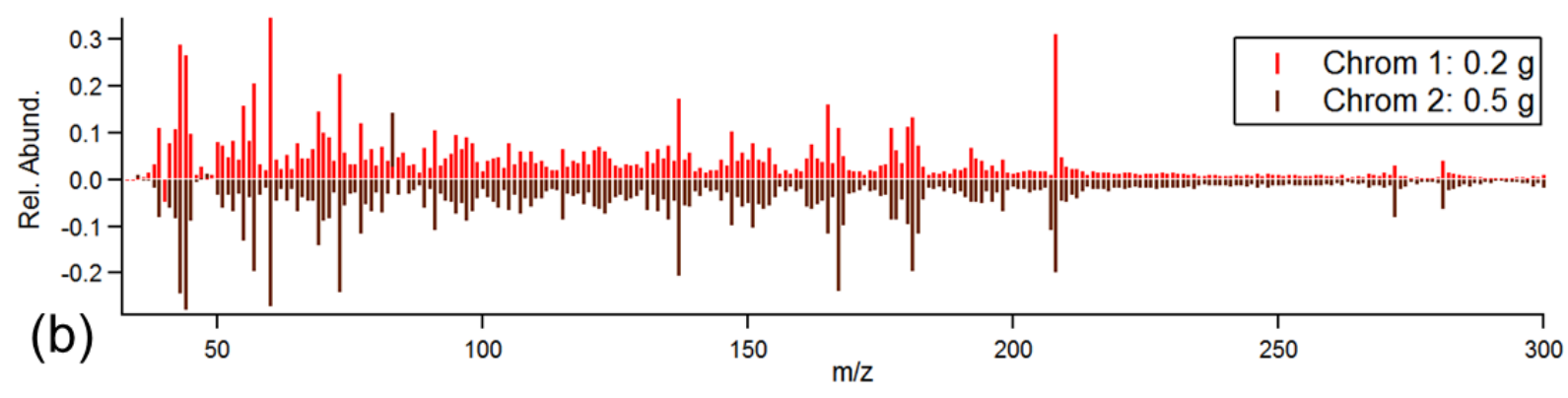

Figure S12. Summed mass spectra for unaged BBOA chromatograms obtained using $0.2 \mathrm{~g}$ ("Chrom 1") and $0.5 \mathrm{~g}$ ("Chrom 2") of: (a) oak leaf, and (b) oak wood. Each mass spectrum was obtained from the chromatogram by subtracting an appropriate system blank, summing all ions across the full chromatogram, and converting the resulting mass spectrum vector to a unit vector per the technique outlined in Stein and Scott (Stein and Scott, 1994). In both plots, the "Chrom 2" unit vector is displayed with a negative relative abundance to facilitate visual comparison of the mass spectra. 


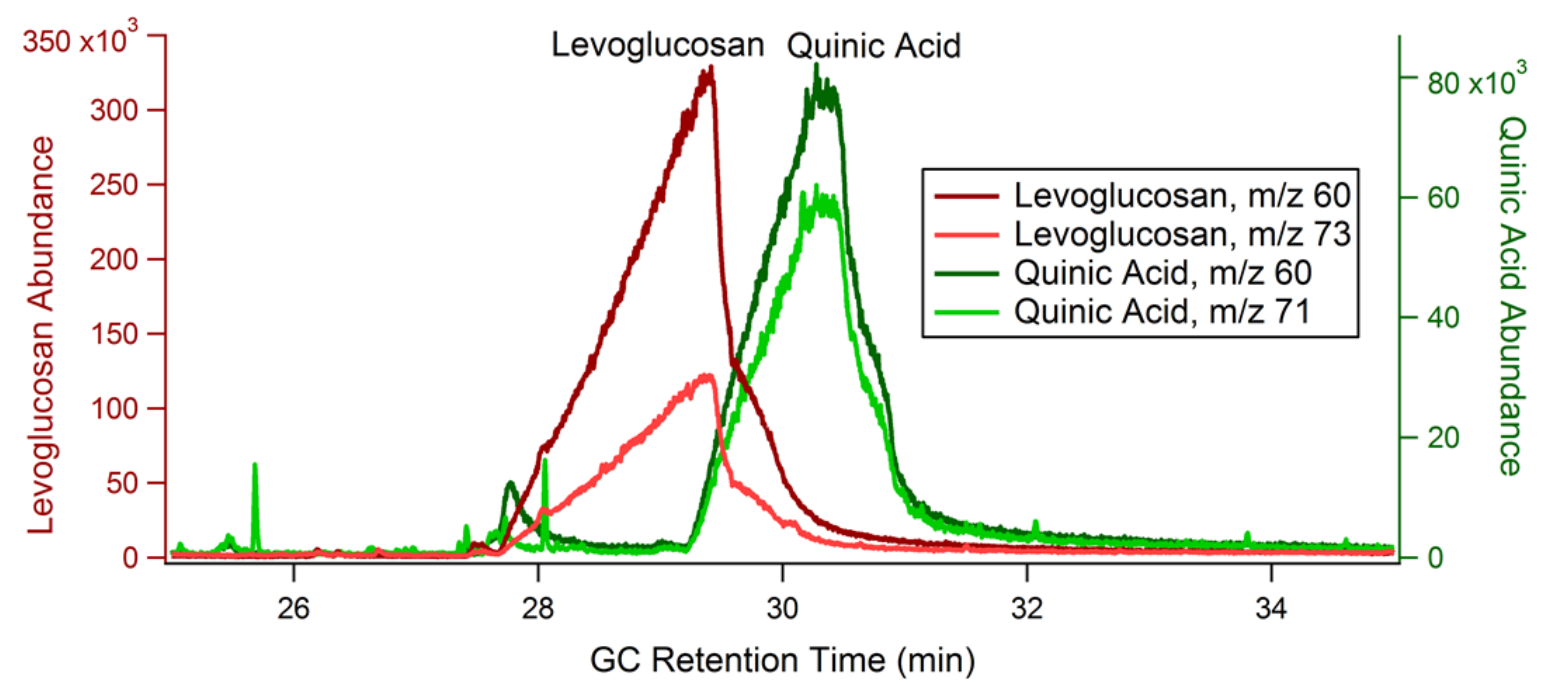

Figure S13. Results from levoglucosan $(15 \mu \mathrm{g})$ and quinic acid $(5 \mu \mathrm{g})$ TAG standard injections. 

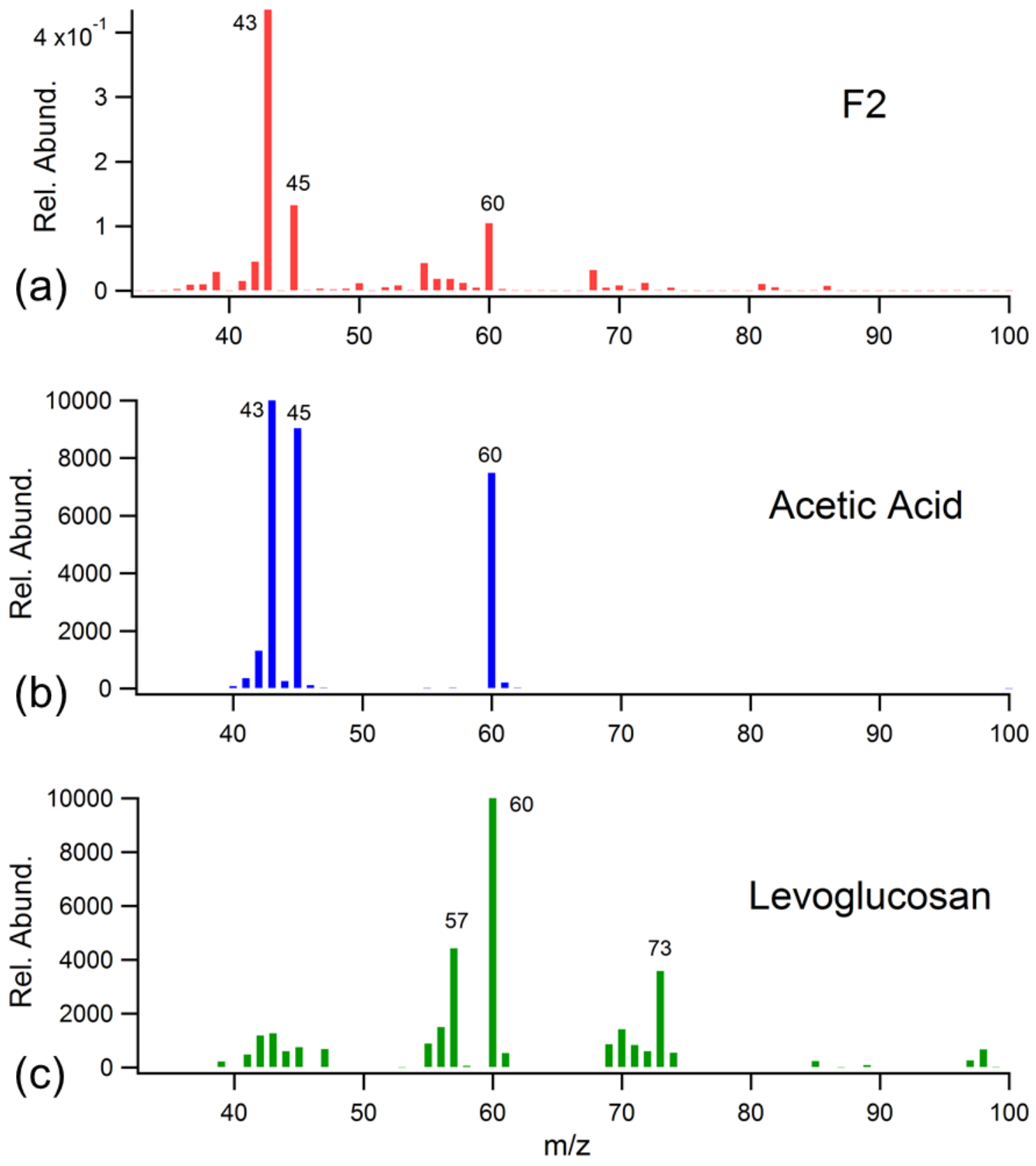

Figure S14. Electron ionization mass spectra for (a) Factor 2 (F2) obtained during oak wood decomposition PMF analysis (Figure 13), (b) Acetic acid (NIST Mass Spec Data Center), and (c) levoglucosan (NIST Mass Spec Data Center). 
Table S1. $\mathrm{OH}_{\text {exp }}$ estimations obtained using the Oxidation Flow Reactor Exposure Estimator version 2.3, available for download at http://sites.google.com/site/pamwiki/hardware/estimation-equations (Peng et al., 2015). Calculations were performed using the OFR185 portion of the spreadsheet. For low and high (1 and $100 \mathrm{~s}^{-1}$, respectively) assumed external $\mathrm{OH}$ reactivity $\left(\mathrm{OHR}_{\text {ext }}\right)$ values, $\mathrm{OH}_{\exp }$ was calculated output using $\mathrm{O}_{3}$ concentrations typical of each level of oxidation ( $\left[\mathrm{O}_{3}\right]$; obtained during reactor calibrations), a reactor residence time of $78 \mathrm{~s}$, and an $\mathrm{RH}$ of $30 \%$. The cells of the table are color-coded to indicate whether the "condition type" was determined to be "safer" (green) or "riskier" (orange).

\begin{tabular}{|c|c|c|c|c|}
\hline \multirow{2}{*}{$\begin{array}{l}\text { Qualitative Level } \\
\text { of Oxidation }^{\mathrm{a}}\end{array}$} & \multirow{2}{*}{$\begin{array}{l}\text { Equiv. Aging Time } \\
\text { (days) }^{\mathrm{a}}\end{array}$} & \multirow[b]{2}{*}[\mathrm{O}_{3}]{$(\mathbf{p p m})$} & \multicolumn{2}{|c|}{ OHexp $\left(\right.$ molec $\left.\mathbf{c m}^{-3} \mathbf{s}\right)$} \\
\hline & & & $O H R_{\text {ext }}=1 \mathrm{~s}^{-1}$ & $O H R_{\text {ext }}=100 \mathrm{~s}^{-1}$ \\
\hline Low-mid & 3.4 & 4.3 & $2.5 \times 10^{12}$ & $3.5 \times 10^{11}$ \\
\hline High & 9.8 & 8.5 & $5.3 \times 10^{12}$ & $1.0 \times 10^{12}$ \\
\hline
\end{tabular}

${ }^{a}$ See Table 1.

Table S2. Maximum SMPS volume concentrations $\left(\mu \mathrm{m}^{3} \mathrm{~cm}^{-3}\right)$ at each level of oxidative aging, presented as an average of triplicate measurements \pm one standard deviation.

\begin{tabular}{|l|l|l|l|}
\hline & \multicolumn{2}{|l|}{ Equivalent Aging Time (days) } & $\mathbf{9 . 8}$ \\
Fuel Type & $\mathbf{0}$ & $\mathbf{3 . 4}$ & $179.04 \pm 28.44$ \\
Leaf & $314.20 \pm 78.59$ & $210.97 \pm 40.82$ & $791.48 \pm 37.61$ \\
Wood & $606.52 \pm 45.32$ & $813.73 \pm 5.61$ & \\
\hline
\end{tabular}


Table S3. Selected compounds of interest identified in oak leaf BBOA TAG chromatograms. Common compound names were used in this work where appropriate and are provided in parentheses. Estimated subcooled liquid vapor pressures $\left(\mathrm{p}_{\mathrm{L}}^{\circ}\right)$, saturation concentrations $\left(\mathrm{C}^{*}\right)$ and corresponding volatility classifications are also provided. Compounds are classified as intermediately volatile, semivolatile, low volatility, or extremely low volatility organic compounds (I-, S-, L-, ELVOCs, respectively) based on the compound's $\log _{10}\left(\mathrm{C}^{*}\right)$ value and criteria outlined in previous literature.

\begin{tabular}{|c|c|c|c|c|c|c|}
\hline Structure & Compound Name & $\begin{array}{l}\text { Certainty } \\
\text { of } \text { ID }^{\mathrm{a}}\end{array}$ & $\begin{array}{l}\text { Molecular } \\
\text { Formula } \\
\end{array}$ & $\begin{array}{l}\text { Predicted } \\
p_{\mathrm{L}}{ }^{\circ} \text { at } 25^{\circ} \mathrm{C} \\
(\text { torr })^{\mathbf{b}}\end{array}$ & $\begin{array}{l}\log _{10}\left(\mathbf{C}^{*}\right) \\
\left(\mu \mathrm{g} \mathrm{m^{-3 }}\right)^{\mathrm{c}}\end{array}$ & $\begin{array}{l}\text { Volatility } \\
\text { Classification } \\
\end{array}$ \\
\hline 1 & $\begin{array}{l}\text { 1,6-anhydro- } \beta \text { - } \\
\text { glucopyranose } \\
\text { (levoglucosan) }\end{array}$ & A & $\mathrm{C}_{6} \mathrm{H}_{10} \mathrm{O}_{5}$ & $1.81 \mathrm{E}-7$ & 0.31 & SVOC \\
\hline 2 & Quinic Acid & A & $\mathrm{C}_{7} \mathrm{H}_{12} \mathrm{O}_{6}$ & $1.58 \mathrm{E}-9$ & -1.67 & LVOC \\
\hline 3 & Mannose & $\mathrm{B}$ & $\mathrm{C}_{16} \mathrm{H}_{12} \mathrm{O}_{6}$ & $2.59 \mathrm{E}-13$ & -5.49 & ELVOC \\
\hline 4 & Octadecanoic Acid & $\mathrm{B}$ & $\mathrm{C}_{18} \mathrm{H}_{36} \mathrm{O}_{2}$ & $8.58 \mathrm{E}-6$ & 2.23 & SVOC \\
\hline 5 & Tricosane ( $\mathrm{C}_{23}$ alkane $)$ & A & $\mathrm{C}_{23} \mathrm{H}_{48}$ & $1.24 \mathrm{E}-05$ & 2.45 & SVOC \\
\hline 6 & Tetracosene ( $\mathrm{C}_{24}$ alkene $)$ & $\mathrm{D}$ & $\mathrm{C}_{24} \mathrm{H}_{48}$ & 8.07E-06 & 2.28 & SVOC \\
\hline 7 & Pentacosane ( $\mathrm{C}_{25}$ alkane $)$ & $\mathrm{A}$ & $\mathrm{C}_{25} \mathrm{H}_{52}$ & $5.90 \mathrm{E}-06$ & 1.82 & SVOC \\
\hline 8 & $\begin{array}{l}\text { Tetracosanal } \\
\left(\mathrm{C}_{24} \text { aldehyde }\right)\end{array}$ & $\mathrm{D}$ & $\mathrm{C}_{24} \mathrm{H}_{48} \mathrm{O}$ & 7.34E-08 & 2.16 & SVOC \\
\hline 9 & Tetracosanol $\left(\mathrm{C}_{24}\right.$ alcohol $)$ & $\mathrm{D}$ & $\mathrm{C}_{24} \mathrm{H}_{50} \mathrm{O}$ & $1.85 \mathrm{E}-06$ & 0.260 & SVOC \\
\hline 10 & $\begin{array}{l}\text { Hexacosanal } \\
\left(\mathrm{C}_{26} \text { aldehyde }\right)\end{array}$ & $\mathrm{D}$ & $\mathrm{C}_{26} \mathrm{H}_{52} \mathrm{O}$ & $1.47 \mathrm{E}-07$ & 1.69 & SVOC \\
\hline 11 & Nonacosane $\left(\mathrm{C}_{29}\right.$ alkane $)$ & A & $\mathrm{C}_{29} \mathrm{H}_{60}$ & $6.18 \mathrm{E}-07$ & 0.623 & SVOC \\
\hline 12 & $\begin{array}{l}\text { Octacosanal } \\
\left(\mathrm{C}_{28} \text { aldehyde }\right)\end{array}$ & $\mathrm{D}$ & $\mathrm{C}_{28} \mathrm{H}_{56} \mathrm{O}$ & 2.19E-07 & 1.25 & SVOC \\
\hline 13 & $\begin{array}{l}\text { Triacontanal } \\
\left(\mathrm{C}_{30} \text { aldehyde }\right)\end{array}$ & $\mathrm{D}$ & $\mathrm{C}_{30} \mathrm{H}_{60} \mathrm{O}$ & $8.22 \mathrm{E}-08$ & 0.825 & SVOC \\
\hline 14 & D:A-Friedoolean-6-ene & B & $\mathrm{C}_{30} \mathrm{H}_{50}$ & $6.15 \mathrm{E}-08$ & 0.247 & SVOC \\
\hline 15 & $\begin{array}{l}\text { Dotriacontanal } \\
\left(\mathrm{C}_{32} \text { aldehyde }\right)\end{array}$ & $\mathrm{D}$ & $\mathrm{C}_{32} \mathrm{H}_{64} \mathrm{O}$ & $2.86 \mathrm{E}-09$ & 0.426 & LVOC \\
\hline 16 & Friedelan-3-one (Friedelin) & B & $\mathrm{C}_{30} \mathrm{H}_{50} \mathrm{O}$ & $1.24 \mathrm{E}-05$ & -1.07 & LVOC \\
\hline
\end{tabular}

a Identification certainty ("Certainty of ID") was classified for each compound according to the following criteria: (A) the compound was positively identified based on external standard injections; (B) the compound was identified based on a high match quality (MQ $>75 \%$ ) using available mass spectral libraries; (C) the compound was identified based on a low-to-moderate match quality ( MQ < 75\%) using available mass spectral libraries; and (D) no adequate mass spectral library match was available for the compound, so the compound structure was determined by retention time and manually evaluating possible fragmentation patterns.

${ }^{\mathrm{b}}$ Vapor pressures at $25^{\circ} \mathrm{C}$ were predicted using the Advanced Chemistry Development (ACD/Labs) Software V11.02 (@ 1994-2017 ACD/Labs) through the SciFinder website (ACD/Labs, 2017).

${ }^{\mathrm{c}} \log _{10}\left(\mathrm{C}^{*}\right)$ values calculated using methods outlined in Pankow, et al. (Pankow, 1994). An activity coefficient of 1.3 was assumed based on a typical estimated BBOA activity coefficient of 1.3 (Donahue et al., 2011).

${ }^{\mathrm{d}}$ Defined using criteria set forth in previous work (Donahue et al., 2011, 2012). 
Table S4. Selected compounds of interest identified in oak wood BBOA TAG chromatograms. Common compound names were used in this work where appropriate and are provided in parentheses. Estimated subcooled liquid vapor pressures $\left(\mathrm{p}_{\mathrm{L}}^{\circ}\right)$, saturation concentrations $\left(\mathrm{C}^{*}\right)$ and corresponding volatility classifications are also provided. Compounds are classified as intermediately volatile, semivolatile, low volatility, or extremely low volatility organic compounds (I-, S-, L-, ELVOCs, respectively) based on the compound's $\log _{10}\left(\mathrm{C}^{*}\right)$ value and criteria outlined in previous literature.

\begin{tabular}{|c|c|c|c|c|c|c|}
\hline Structure & Compound Name & $\begin{array}{l}\text { Certainty } \\
\text { of ID }\end{array}$ & $\begin{array}{l}\text { Molecular } \\
\text { Formula } \\
\end{array}$ & $\begin{array}{l}\text { Predicted } \\
p_{L}{ }^{{ }^{b}}\end{array}$ & $\begin{array}{l}\log _{10}\left(C^{*}\right) \\
\left(\mu \mathrm{g} \mathrm{m}^{-3}\right)^{\mathrm{c}} \\
\end{array}$ & $\begin{array}{l}\text { Volatility } \\
\text { Classification }^{\mathrm{d}} \\
\end{array}$ \\
\hline 17 & $\begin{array}{l}\text { 2,6-dimethoxy-phenol } \\
\text { (syringol) }\end{array}$ & $\mathrm{B}$ & $\mathrm{C}_{8} \mathrm{H}_{10} \mathrm{O}_{3}$ & $5.91 \mathrm{E}-3$ & 4.80 & IVOC \\
\hline 18 & $\begin{array}{l}\text { 4-hydroxy-3-methoxy- } \\
\text { benzaldehyde (vanillin) }\end{array}$ & B & $\mathrm{C}_{8} \mathrm{H}_{8} \mathrm{O}_{3}$ & $1.94 \mathrm{E}-3$ & 4.31 & IVOC \\
\hline 19 & $\begin{array}{l}\text { 4-(1,2-propadienyl)- } \\
\text { guaiacol }\end{array}$ & B & $\mathrm{C}_{10} \mathrm{H}_{10} \mathrm{O}_{2}$ & $1.16 \mathrm{E}-3$ & 4.12 & IVOC \\
\hline 20 & $\begin{array}{l}\text { 1,6-anhydro- } \beta \text { - } \\
\text { glucopyranose } \\
\text { (levoglucosan) }\end{array}$ & A & $\mathrm{C}_{6} \mathrm{H}_{10} \mathrm{O}_{5}$ & $1.81 \mathrm{E}-7$ & 0.31 & SVOC \\
\hline 21 & galacto-heptulose & $\mathrm{C}$ & $\mathrm{C}_{7} \mathrm{H}_{14} \mathrm{O}_{7}$ & $2.08 \mathrm{E}-18$ & -10.51 & ELVOC \\
\hline 22 & $\begin{array}{l}\text { 1,6-anhydro- } \alpha \text {-d- } \\
\text { galactofuranose }\end{array}$ & $\mathrm{C}$ & $\mathrm{C}_{6} \mathrm{H}_{10} \mathrm{O}_{5}$ & $1.12 \mathrm{E}-8$ & -0.896 & LVOC \\
\hline 23 & guanosine & $\mathrm{C}$ & $\mathrm{C}_{10} \mathrm{H}_{13} \mathrm{~N}_{5} \mathrm{O}_{5}$ & $2.44 \mathrm{E}-25$ & -17.32 & ELVOC \\
\hline 24 & n-acetyl-d-galactosamine & $\mathrm{C}$ & $\mathrm{C}_{8} \mathrm{H}_{15} \mathrm{NO}_{6}$ & 7.12E-9 & -10.96 & ELVOC \\
\hline 25 & $\begin{array}{l}\text { 2,6-di-methoxy-4-vinyl- } \\
\text { phenol } \\
\text { (4-vinyl-syringol) }\end{array}$ & B & $\mathrm{C}_{10} \mathrm{H}_{12} \mathrm{O}_{3}$ & $1.63 \mathrm{E}-3$ & 4.31 & IVOC \\
\hline 26 & $\begin{array}{l}\text { 4-hydroxy-3,5-dimethoxy- } \\
\text { benzaldehyde } \\
\text { (syringaldehyde) }\end{array}$ & B & $\mathrm{C}_{9} \mathrm{H}_{10} \mathrm{O}_{4}$ & $1.51 \mathrm{E}-4$ & 3.28 & IVOC \\
\hline 27 & $\begin{array}{l}\text { 2,6-dimethoxy-4-(1- } \\
\text { propynyl)-phenol }\end{array}$ & $\mathrm{C}$ & $\mathrm{C}_{11} \mathrm{H}_{12} \mathrm{O}_{3}$ & $8.95 \mathrm{E}-5$ & 3.08 & IVOC \\
\hline 28 & $\begin{array}{l}\text { 1-(4-hydroxy-3,5- } \\
\text { dimethoxyphenyl)-ethanone } \\
\text { (acetosyringone) }\end{array}$ & B & $\mathrm{C}_{10} \mathrm{H}_{12} \mathrm{O}_{4}$ & $6.49 \mathrm{E}-5$ & 2.95 & IVOC \\
\hline 29 & $\begin{array}{l}\text { 3-(4-hydroxy-3- } \\
\text { methoxyphenyl)prop-2-enal } \\
\text { (coniferaldehyde) }\end{array}$ & B & $\mathrm{C}_{10} \mathrm{H}_{10} \mathrm{O}_{3}$ & $4.88 \mathrm{E}-5$ & 2.78 & IVOC \\
\hline 30 & $\begin{array}{l}\text { 4-(2-oxopropyl)-syringol } \\
\text { (syringyl acetone) }\end{array}$ & B & $\mathrm{C}_{11} \mathrm{H}_{14} \mathrm{O}_{4}$ & $4.49 \mathrm{E}-5$ & 2.82 & IVOC \\
\hline 31 & $\begin{array}{l}\text { 4-hydroxy-3,5- } \\
\text { dimethoxycinnamaldehyde } \\
\text { (sinapaldehyde) }\end{array}$ & B & $\mathrm{C}_{11} \mathrm{H}_{12} \mathrm{O}_{4}$ & $4.53 \mathrm{E}-6$ & 1.82 & SVOC \\
\hline
\end{tabular}

a Identification certainty ("Certainty of ID") was classified for each compound according to the following criteria: (A) the compound was positively identified based on external standard injections; (B) the compound was identified based on a high match quality (MQ $>75 \%$ ) using available mass spectral libraries; (C) the compound was identified based on a low-to-moderate match quality $(50 \%<\mathrm{MQ}<75 \%)$ using available mass spectral libraries; and (D) no adequate mass spectral library match was available for the compound, so the compound structure was determined by retention time and manually evaluating possible fragmentation patterns.

${ }^{\mathrm{b}}$ Vapor pressures at $25^{\circ} \mathrm{C}$ were predicted using the Advanced Chemistry Development (ACD/Labs) Software V11.02 (@ 1994-2017 ACD/Labs) through the SciFinder website (ACD/Labs, 2017).

${ }^{\mathrm{c}} \log _{10}\left(\mathrm{C}^{*}\right)$ values calculated using methods outlined in Pankow, et al. (Pankow, 1994). An activity coefficient of 1.3 was assumed based on a typical estimated BBOA activity coefficient of 1.3 (Donahue et al., 2011).

${ }^{\mathrm{d}}$ Defined using criteria set forth in previous work (Donahue et al., 2011, 2012). 
Table S5. Raw SIC integrations, reported as a triplicate average \pm one standard deviation (\%), for each compound reported in Table S2 at each equivalent aging time.

\begin{tabular}{|c|c|c|c|c|}
\hline Compound & $\begin{array}{l}\text { Ion } \\
\text { Integrated }\end{array}$ & Equivalent Aging & ime (days) & 9.8 \\
\hline $\begin{array}{l}\text { 1,6-anhydro- } \beta \text {-glucopyranose } \\
\text { (levoglucosan) }\end{array}$ & 60 & $1808457 \pm 81.0 \%$ & $204658 \pm 105 \%$ & $30721 \pm 82.8 \%$ \\
\hline Quinic Acid & 60 & $11018541 \pm 58.1 \%$ & $1853570 \pm 95.0 \%$ & $563580 \pm 100 \%$ \\
\hline Mannose & 60 & $1251912 \pm 69.5 \%$ & $50679 \pm 85.7$ & $3009 \pm 72.7 \%$ \\
\hline Octadecanoic Acid & 60 & $152898 \pm 84.2 \%$ & $9662 \pm 44.4 \%$ & $0 *$ \\
\hline Tricosane ( $\mathrm{C}_{23}$ alkane $)$ & 57 & $1436688 \pm 40.5 \%$ & $586016 \pm 18.4 \%$ & $333239 \pm 26.7 \%$ \\
\hline Pentacosane ( $\mathrm{C}_{25}$ alkane $)$ & 57 & $2906372 \pm 50.0 \%$ & $1058663 \pm 21.9 \%$ & $436707 \pm 35.6 \%$ \\
\hline Tetracosanal $\left(\mathrm{C}_{24}\right.$ aldehyde $)$ & 82 & $5677688 \pm 28.5 \%$ & $2827439 \pm 23.9 \%$ & $662488 \pm 133 \%$ \\
\hline Tetracosanol $\left(\mathrm{C}_{24}\right.$ alcohol $)$ & 97 & $25287834 \pm 40.7 \%$ & $7149720 \pm 52.5 \%$ & $1668500 \pm 91.4 \%$ \\
\hline Hexacosanal $\left(\mathrm{C}_{26}\right.$ aldehyde $)$ & 82 & $4848128 \pm 52.5$ & $816648 \pm 51.9 \%$ & $123991 \pm 100 \%$ \\
\hline Nonacosane $\left(\mathrm{C}_{29}\right.$ alkane $)$ & 57 & $5239775 \pm 47.4 \%$ & $1999041 \pm 22.8 \%$ & $754266 \pm 43.8 \%$ \\
\hline Octacosanal ( $\mathrm{C}_{28}$ aldehyde) & 82 & $5289335 \pm 60.5 \%$ & $469549 \pm 74.3 \%$ & $37070 \pm 105 \%$ \\
\hline Triacontanal $\left(\mathrm{C}_{30}\right.$ aldehyde $)$ & 82 & $2726165 \pm 73.7 \%$ & $79372 \pm 76.1 \%$ & $3974 \pm 141 \%$ \\
\hline Dotriacontanal ( $\mathrm{C}_{32}$ aldehyde) & 82 & $624787 \pm 81.1 \%$ & $0 *$ & $0 *$ \\
\hline D:A-Friedoolean-6-ene & 95 & $1579365 \pm 37.8 \%$ & $625577 \pm 30.1 \%$ & $220015 \pm 60.3 \%$ \\
\hline Friedelan-3-one (Friedelin) & 95 & $1691654 \pm 53.5 \%$ & $316440 \pm 51.7 \%$ & $73358 \pm 75.3 \%$ \\
\hline
\end{tabular}

*Signal not present above noise in any of the triplicate chromatograms. 
Table S6. Raw SIC integrations, reported as a triplicate average \pm one standard deviation (\%), for each compound reported in Table S3 at each equivalent aging time.

\begin{tabular}{|c|c|c|c|c|}
\hline Compound & $\begin{array}{l}\text { Ion } \\
\text { Integrated }\end{array}$ & $\begin{array}{l}\text { Equivalent Aging } 1 \\
\text { 0 }\end{array}$ & ime (days) & 9.8 \\
\hline 2,6-dimethoxy-phenol (syringol) & 154 & $1201740 \pm 10.2 \%$ & $3010168 \pm 5.56 \%$ & $1336464 \pm 4.77 \%$ \\
\hline $\begin{array}{l}\text { 4-hydroxy-3-methoxy- } \\
\text { benzaldehyde (vanillin) }\end{array}$ & 152 & $507509 \pm 22.5 \%$ & $6109405 \pm 5.27 \%$ & $1377979 \pm 16.5 \%$ \\
\hline 4-(1,2-propadienyl)-guaiacol & 162 & $1993611 \pm 12.5 \%$ & $150577 \pm 9.07 \%$ & $20772 \pm 12.1 \%$ \\
\hline $\begin{array}{l}\text { 1,6-anhydro- } \beta \text {-glucopyranose } \\
\text { (levoglucosan) }\end{array}$ & 60 & $18902529 \pm 6.04 \%$ & $24208958 \pm 11.0 \%$ & $4198164 \pm 12.5 \%$ \\
\hline galacto-heptulose & 60 & $558671 \pm 27.6 \%$ & $1066469 \pm 21.6 \%$ & $155169 \pm 3.62 \%$ \\
\hline 1,6-anhydro- $\alpha$-d-galactofuranose & 60 & $74564 \pm 10.2 \%$ & $107780 \pm 14.0 \%$ & $0^{*}$ \\
\hline guanosine & 60 & $79398 \pm 7.43 \%$ & $105568 \pm 1.78 \%$ & $21293 \pm 0.491 \%$ \\
\hline n-acetyl-d-galactosamine & 60 & $152367 \pm 10.9 \%$ & $205321 \pm 7.25 \%$ & $50226 \pm 5.28$ \\
\hline $\begin{array}{l}\text { 2,6-di-methoxy-4-vinyl-phenol } \\
\text { (4-vinyl-syringol) }\end{array}$ & 180 & $987072 \pm 3.72 \%$ & $764852 \pm 2.06$ & $302326 \pm 9.09 \%$ \\
\hline $\begin{array}{l}\text { 4-hydroxy-3,5-dimethoxy- } \\
\text { benzaldehyde (syringaldehyde) }\end{array}$ & 182 & $2918961 \pm 13.8 \%$ & $8383320 \pm 1.87 \%$ & $2704184 \pm 13.3 \%$ \\
\hline $\begin{array}{l}\text { 2,6-dimethoxy-4-(1-propynyl)- } \\
\text { phenol }\end{array}$ & 192 & $2291745 \pm 11.3 \%$ & $93509 \pm 10.5 \%$ & $12448 \pm 20.8 \%$ \\
\hline $\begin{array}{l}\text { 1-(4-hydroxy-3,5- } \\
\text { dimethoxyphenyl)-ethanone } \\
\text { (acetosyringone) }\end{array}$ & 181 & $1893305 \pm 9.62 \%$ & $1374845 \pm 1.85 \%$ & $559574 \pm 9.48 \%$ \\
\hline $\begin{array}{l}\text { 3-(4-hydroxy-3- } \\
\text { methoxyphenyl)prop-2-enal } \\
\text { (coniferaldehyde) }\end{array}$ & 178 & $2229631 \pm 25.8 \%$ & $874140 \pm 5.91 \%$ & $88980 \pm 23.5 \%$ \\
\hline $\begin{array}{l}\text { 4-(2-oxopropyl)-syringol } \\
\text { (syringyl acetone) }\end{array}$ & 210 & $2611809 \pm 13.6 \%$ & $1364254 \pm 6.01 \%$ & $165647 \pm 22.3 \%$ \\
\hline $\begin{array}{l}\text { 4-hydroxy-2- } \\
\text { methoxycinnamaldehyde } \\
\text { (sinapaldehyde) }\end{array}$ & 208 & $20025099 \pm 4.07 \%$ & $8043741 \pm 1.18 \%$ & $1467539 \pm 13.1 \%$ \\
\hline
\end{tabular}

*Signal not present above noise in any of the triplicate chromatograms.

Table S7. Dot products of mass spectral vectors obtained from TAG chromatograms collected using two different fuel masses at each equivalent aging time. A dot product of 1 signifies a perfect mass spectral match, and a dot product of 0 indicates a complete mismatch (Stein and Scott, 1994).

\begin{tabular}{|l|l|l|l|}
\hline & \multicolumn{2}{l|}{} \\
Fuel Type & $\mathbf{0}$ & $\mathbf{3 . 4}$ & $\mathbf{9 . 8}$ \\
\hline Leaf & 0.847 & 0.764 & 0.787 \\
Wood & 0.934 & 0.784 & 0.843 \\
\hline
\end{tabular}


Table S8. Relative abundances of integrated compounds displayed in Figure 20, presented as triplicate-averaged percentages of the total compound window $m / z 60$ signal.

\begin{tabular}{|l|l|l|l|l|}
\hline \multirow{5}{*}{ Fuel Type } & \multicolumn{5}{|l|}{ Equivalent Aging Time (days) } \\
\hline \multirow{5}{*}{ Leaf } & Compound & $\mathbf{0}$ & $\mathbf{3 . 4}$ & $\mathbf{9 . 8}$ \\
\hline & levoglucosan & $8.35 \pm 3.61 \%$ & $4.92 \pm 1.69 \%$ & $3.20 \pm 0.0699 \%^{\mathrm{a}}$ \\
& quinic acid & $60.7 \pm 5.50 \%$ & $44.5 \pm 16.1 \%$ & $48.8 \pm 7.86 \%^{\mathrm{a}}$ \\
& mannose & $5.00 \pm 2.89 \%$ & $1.43 \pm 0.122 \%$ & $0.341 \pm 0.0831 \%^{\mathrm{a}}$ \\
& octadecanoic acid & $0.698 \pm 0.396 \%$ & $0.405 \pm 0.182 \%$ & $0^{\mathrm{b}}$ \\
\hline \multirow{5}{*}{ Wood } & guanosine & $0.348 \pm 0.0344 \%$ & $0.364 \pm 0.0429 \%$ & $0.363 \pm 0.0229 \%$ \\
& levoglucosan & $82.4 \pm 1.36 \%$ & $82.3 \pm 0.285 \%$ & $70.7 \pm 1.30 \%$ \\
& $\begin{array}{l}\text { galacto-heptulose } \\
\text { n-acetyl-d- }\end{array}$ & $2.42 \pm 0.590 \%$ & $3.59 \pm 0.369 \%$ & $2.64 \pm 0.0849 \%$ \\
& galactosamine & $0.663 \pm 0.0457 \%$ & $0.702 \pm 0.0588 \%$ & $0.851 \pm 0.0248 \%$ \\
& 1,6-anhydro- $\alpha-d-$ & $0.325 \pm 0.0216 \%$ & $0.367 \pm 0.0358 \%$ & $0^{\mathrm{b}}$ \\
\hline
\end{tabular}

a Signal only present above noise in two of three triplicate chromatograms; values are therefore obtained using the two chromatograms with sufficient signal.

${ }^{\mathrm{b}}$ Signal not present above noise in any of the triplicate chromatograms.

\section{References.}

ACD/Labs: Advanced Chemistry Development (ACD/Labs) Software V11.02 (@ 1994-2012 ACD/Labs), Retrieved from http: //www.cas.org/products/scifinder, 11 May, 2017.

Davis, D. D., Ravishankara, A. R. and Fischer, S.: SO2 oxidation via the hydroxyl radical: Atmospheric fate of HSOx radicals, Geophys. Res. Lett., 6(2), 113-116, doi:10.1029/GL006i002p00113, 1979.

Donahue, N. M., Epstein, S. A., Pandis, S. N. and Robinson, A. L.: A two-dimensional volatility basis set: 1. organicaerosol mixing thermodynamics, Atmos Chem Phys, 11(7), 3303-3318, doi:10.5194/acp-11-3303-2011, 2011.

Donahue, N. M., Kroll, J. H., Pandis, S. N. and Robinson, A. L.: A two-dimensional volatility basis set - Part 2: Diagnostics of organic-aerosol evolution, Atmos Chem Phys, 12(2), 615-634, doi:10.5194/acp-12-615-2012, 2012.

Ehhalt, D. H., Dorn, H.-P. and Poppe, D.: The chemistry of the hydroxyl radical in the troposphere, Proc. R. Soc. Edinb. Sect. B Biol. Sci., 97, 17-34, doi:10.1017/S0269727000005273, 1990.

Gulz, P. G. and Boor, G.: Seasonal Variations in Epicuticular Wax Ultrastructures of Quercus robur Leaves, Z Naturforsch, 47c, 807-814, 1992.

Kang, E., Root, M. J., Toohey, D. W. and Brune, W. H.: Introducing the concept of Potential Aerosol Mass (PAM), Atmos Chem Phys, 7(22), 5727-5744, doi:10.5194/acp-7-5727-2007, 2007.

Kreisberg, N. M., Hering, S. V., Williams, B. J., Worton, D. R. and Goldstein, A. H.: Quantification of Hourly Speciated Organic Compounds in Atmospheric Aerosols, Measured by an In-Situ Thermal Desorption Aerosol Gas Chromatograph (TAG), Aerosol Sci. Technol., 43(1), 38-52, doi:10.1080/02786820802459583, 2009.

Lambe, A. T., Ahern, A. T., Williams, L. R., Slowik, J. G., Wong, J. P. S., Abbatt, J. P. D., Brune, W. H., Ng, N. L., Wright, J. P., Croasdale, D. R., Worsnop, D. R., Davidovits, P. and Onasch, T. B.: Characterization of aerosol photooxidation flow reactors: heterogeneous oxidation, secondary organic aerosol formation and cloud condensation nuclei activity measurements, Atmos Meas Tech, 4(3), 445-461, doi:10.5194/amt-4-445-2011, 2011. 
Levy, H.: Normal Atmosphere: Large Radical and Formaldehyde Concentrations Predicted, Science, 173(3992), 141$143,1971$.

Li, R., Palm, B. B., Ortega, A. M., Hlywiak, J., Hu, W., Peng, Z., Day, D. A., Knote, C., Brune, W. H., de Gouw, J. A. and Jimenez, J. L.: Modeling the Radical Chemistry in an Oxidation Flow Reactor: Radical Formation and Recycling, Sensitivities, and the OH Exposure Estimation Equation, J. Phys. Chem. A, 119(19), 4418-4432, doi:10.1021/jp509534k, 2015.

Mao, J., Ren, X., Brune, W. H., Olson, J. R., Crawford, J. H., Fried, A., Huey, L. G., Cohen, R. C., Heikes, B., Singh, H. B., Blake, D. R., Sachse, G. W., Diskin, G. S., Hall, S. R. and Shetter, R. E.: Airborne measurement of OH reactivity during INTEX-B, Atmos Chem Phys, 9(1), 163-173, doi:10.5194/acp-9-163-2009, 2009.

NIST Mass Spec Data Center, S.E. Stein, director "Mass Spectra" in NIST Chemistry WebBook, NIST Standard Reference Database Number 69, National Institute of Standards and Technology, Gaithersburg MD, 20899, retrieved 4 June, 2017.

Palm, B. B., Campuzano-Jost, P., Ortega, A. M., Day, D. A., Kaser, L., Jud, W., Karl, T., Hansel, A., Hunter, J. F., Cross, E. S., Kroll, J. H., Peng, Z., Brune, W. H. and Jimenez, J. L.: In situ secondary organic aerosol formation from ambient pine forest air using an oxidation flow reactor, Atmos Chem Phys, 16(5), 2943-2970, doi:10.5194/acp-162943-2016, 2016.

Pankow, J. F.: An absorption model of gas/particle partitioning of organic compounds in the atmosphere, Atmos. Environ., 28(2), 185-188, doi:10.1016/1352-2310(94)90093-0, 1994.

Peng, Z., Day, D. A., Stark, H., Li, R., Lee-Taylor, J., Palm, B. B., Brune, W. H. and Jimenez, J. L.: HOx radical chemistry in oxidation flow reactors with low-pressure mercury lamps systematically examined by modeling, Atmos Meas Tech, 8(11), 4863-4890, doi:10.5194/amt-8-4863-2015, 2015.

Peng, Z., Day, D. A., Ortega, A. M., Palm, B. B., Hu, W., Stark, H., Li, R., Tsigaridis, K., Brune, W. H. and Jimenez, J. L.: Non-OH chemistry in oxidation flow reactors for the study of atmospheric chemistry systematically examined by modeling, Atmos Chem Phys, 16(7), 4283-4305, doi:10.5194/acp-16-4283-2016, 2016.

Shetter, R. E., Cantrell, C. A., Lantz, K. O., Flocke, S. J., Orlando, J. J., Tyndall, G. S., Gilpin, T. M., Fischer, C. A., Madronich, S., Calvert, J. G. and Junkermann, W.: Actinometric and radiometric measurement and modeling of the photolysis rate coefficient of ozone to O(1D) during Mauna Loa Observatory Photochemistry Experiment 2, J. Geophys. Res. Atmospheres, 101(D9), 14631-14642, doi:10.1029/96JD00211, 1996.

Stein, S. E. and Scott, D. R.: Optimization and testing of mass spectral library search algorithms for compound identification, J. Am. Soc. Mass Spectrom., 5(9), 859-866, doi:10.1016/1044-0305(94)87009-8, 1994.

Wong, Z., Chen, K. and Li, J.: Formation of Vanillin and Syringaldehyde in an Oxygen Delignification Process, BioResources, 5(3), 1509-1516, doi:10.15376/biores.5.3.1509-1516, 2010. 